\title{
A property of Hilbert curves of scrolls over surfaces
}

\author{
Antonio Lanteri
}

\begin{abstract}
Let $(X, L)$ be a polarized manifold of dimension $n$. Its Hilbert curve is an affine algebraic plane curve of degree $n$ encoding properties related to fibrations of $X$, defined by suitable adjoint linear systems to $L$. In particular, if $(X, L)$ is a scroll over a smooth surface $S$, its Hilbert curve consists of $n-2$ parallel lines with a given slope and evenly spaced, plus a conic. Making its equation explicit, we show that this conic turns out to be itself the Hilbert curve of the $\mathbb{Q}$-polarized surface $\left(S, \frac{1}{n-1} \operatorname{det} \mathcal{E}\right)$, where $\mathcal{E}$ is the rank- $(n-1)$ vector bundle obtained by pushing down $L$ via the scroll projection, if and only if $\mathcal{E}$ is properly semistable in the sense of Bogomolov.
\end{abstract}

2010 Mathematics Subject Classification. Primary 14C20; Secondary 14J60, 14N30.

Keywords and phrases. Scroll; Hilbert curve; vector bundle (properly semistable); $\mathbb{Q}$-polarized surface.

\section{Introduction}

The Hilbert curve $\Gamma$ of a polarized manifold $(X, L)$ was introduced in [4]. It is an algebraic affine plane curve of degree $n=\operatorname{dim} X$, encoding several properties of $(X, L)$. In particular it is sensitive to the possibility of fibering $X$ over a variety of smaller dimension via an adjoint bundle to $L$. This makes scrolls very interesting from the point of view of their Hilbert curves. Scrolls over a curve are discussed in [7]. Here we focus on scrolls over a surface $S$ in any dimension (for the 3 -dimensional case we refer to [9]). In this case, $\Gamma$ consists of $n-2$ parallel lines with a given slope and evenly spaced, plus a conic, say $G$. It should be emphasized that, in general, there is no $\mathbb{Q}$-polarized surface admitting $G$ as Hilbert curve. However, it looks natural to ask whether, in some specific framework, $G$ is itself the Hilbert curve of the base surface $S$ of $(X, L)$ for some $\mathbb{Q}$-polarization related to the scroll [4, Problem 6.6]. To answer this question, we need first to determine the equation of $\Gamma$. To do that, unlike in [9], we skip the explicit expression of $\chi\left(x K_{X}+y L\right)$ provided by the Riemann-Roch theorem, confining ourselves to use the qualitative information coming from [4, Theorem 6.5] combined with the analysis of the homogeneous polynomial it defines, when restricted to the line at infinity of the $(x, y)$-plane. In this way, computing very few pluridegrees of $(X, L)$ turns out to be enough to obtain all coefficients of the polynomial we need (Theorem 3.1). In particular we get the explicit equation of the conic $G$. This allows us to address the above question, extending the main result of $[9]$. In fact we show that $G$ itself is the Hilbert curve of the $\mathbb{Q}$-polarized surface $\left(S, \frac{1}{n-1} \operatorname{det} \mathcal{E}\right)$, where $\mathcal{E}$ is the rank- $(n-1)$ vector bundle obtained by pushing down $L$ via the scroll projection, if and only if $\mathcal{E}$ is properly semistable in the sense of Bogomolov (Theorem 4.1). The case when $\mathcal{E}$ is not properly semistable is also explored. This leads to a number of necessary conditions for $G$ to be the Hilbert curve of the base surface $S$ for some $\mathbb{Q}$-polarization (Proposition 4.4). 


\section{Background material}

Varieties considered in this paper are defined over the field $\mathbb{C}$ of complex numbers. We use the standard notation and terminology from algebraic geometry. A manifold is any smooth projective variety; a surface is a manifold of dimension 2 . The symbol $\equiv$ will denote numerical equivalence. With a little abuse, we adopt the additive notation for the tensor products of line bundles. A polarized manifold is a pair $(X, L)$ consisting of a manifold $X$ endowed with an ample line bundle $L$. In particular, the word scroll has to be intended in the classical sense: since we are dealing with scrolls over a surface $S$, this means that $X=\mathbb{P}_{S}(\mathcal{V})$, where $\mathcal{V}$ is an ample vector bundle or rank $r \geq 2$ on $S$ and $L$ is the corresponding tautological line bundle. Notice that such an object is also a scroll in the adjunction theoretic sense except for very few cases, see [3, Theorem 2.1].

For the notion and the general properties of the Hilbert curve associated to a polarized manifold we refer to [4], see also [7]. Here we just recall some basic facts. Let $(X, L)$ be a polarized manifold of dimension $n \geq 2$ and regard $\mathrm{N}(X):=\operatorname{Num}(X) \otimes_{\mathbb{Z}} \mathbb{C}$ as a complex affine space. If $\operatorname{rk}\left\langle K_{X}, L\right\rangle=2$, we can consider the plane $\mathbb{A}^{2}=\mathbb{C}\left\langle K_{X}, L\right\rangle \subset \mathrm{N}(X)$, generated by the classes of $K_{X}$ and $L$. For any line bundle $D$ on $X$ the Riemann-Roch theorem provides an expression for the Euler-Poincaré characteristic $\chi(D)$ in terms of $D$ and the Chern classes of $X$. Let $p$ denote the complexified polynomial of $\chi(D)$, when we set $D=x K_{X}+y L$, with $x, y$ complex numbers, namely $p(x, y)=\chi\left(x K_{X}+y L\right)$. The Hilbert curve of $(X, L)$ is the complex affine plane curve $\Gamma=\Gamma_{(X, L)} \subset \mathbb{A}^{2}$ of degree $n$ defined by $p(x, y)=0$ [4, Section 2]. Sometimes, to deal with points at infinity, it is convenient to consider also the projective Hilbert curve $\bar{\Gamma} \subset \mathbb{P}^{2}$, namely the projective closure of $\Gamma$. In this case we use $(x, y, z)$ as homogeneous coordinates on $\mathbb{P}^{2}, z=0$ representing the line at infinity.

Notice that the Hilbert curve can be defined also when the numerical classes of $K_{X}$ and $L$ are linearly dependent, but in this case, the $(x, y)$-plane is only formal and $\Gamma_{(X, L)}$ loses the meaning of a plane section of the Hilbert variety of $X$ (see [4, Section 2]). For example, the Hilbert curve of $\left(\mathbb{P}^{n}, \mathcal{O}_{\mathbb{P}^{n}}(r)\right)$ has equation $p(x, y)=\frac{(-1)^{n}}{n !} \prod_{i=1}^{n}((n+1) x-r y-i)$.

Due to Serre duality, $\Gamma$ is invariant under the involution $D \mapsto K_{X}-D$ acting on $\mathrm{N}(X)$. Thus, to make this symmetry more evident, it is convenient to represent $\Gamma$ in terms of the affine coordinates $\left(u=x-\frac{1}{2}, v=y\right)$ rather than $(x, y)$. So, rewriting our divisor as $D=\frac{1}{2} K_{X}+E$, where $E=u K_{X}+v L, \Gamma$ can be represented with respect to these coordinates by $p\left(\frac{1}{2}+u, v\right)=0$. We refer to this equation as the canonical equation of $\Gamma$.

In particular the canonical equation of the Hilbert curve $\Gamma_{(S, \mathcal{L})}$ of a polarized surface $(S, \mathcal{L})$ is:

$$
p_{(S, \mathcal{L})}\left(\frac{1}{2}+u, v\right)=\frac{1}{2}\left[\left(u K_{S}+v \mathcal{L}\right)^{2}+2 \chi\left(\mathcal{O}_{S}\right)-\frac{1}{4} K_{S}^{2}\right]=0 .
$$

Now, let $\mathcal{L}$ is an ample $\mathbb{Q}$-line bundle on the surface $S$. Then there exists a positive integer $m$ such that $\mathcal{M}:=m \mathcal{L} \in \operatorname{Pic}(S)$. Letting $p_{(S, \mathcal{L})}\left(\frac{1}{2}+u, v\right)$ denote the extension of the polynomial expression $\chi\left(\frac{1}{2} K_{S}+E\right)$ where $E=u K_{S}+v \mathcal{L}$, from the equality $E=u K_{S}+\frac{v}{m} \mathcal{M}$ we see that $p_{(S, \mathcal{L})}\left(\frac{1}{2}+u, v\right)=p_{(S, \mathcal{M})}\left(\frac{1}{2}+u, \frac{v}{m}\right)$, the polynomial defining the canonical equation of the Hilbert curve $\Gamma_{(S, \mathcal{M})}$. Thus we can speak about the Hilbert curve $\Gamma_{(S, \mathcal{L})}$ of the $\mathbb{Q}$-polarized surface $(S, \mathcal{L})$, its canonical equation being formally the same equation as (1). 
Extending the terminology in [8], we say that two ample $\mathbb{Q}$-line bundles $\mathcal{L}$ and $\mathcal{L}^{\prime}$ on the surface $S$ are $H C$ equivalent if $\Gamma_{(S, \mathcal{L})}=\Gamma_{\left(S, \mathcal{L}^{\prime}\right)}$. Clearly numerical equivalence implies HC-equivalence, and this, in turn, implies that $\mathcal{L}^{2}=\mathcal{L}^{\prime 2}$ and $K_{S} \cdot \mathcal{L}=K_{S} \cdot \mathcal{L}^{\prime}$, provided that $\left(K_{S}^{2}, \chi\left(\mathcal{O}_{S}\right)\right) \neq(0,0)[8$, Proposition 2.1]. Finally, if $\mathcal{E}$ is an ample vector bundle of rank $r \geq 2$ on $S$, by average polarization induced by $\mathcal{E}$ we mean the ample $\mathbb{Q}$-line bundle $\frac{1}{r} \operatorname{det} \mathcal{E}$.

For any vector bundle $\mathcal{V}$ of rank $r \geq 2$ on a surface $S$, the Bogomolov number of $\mathcal{V}$ is

$$
\delta(\mathcal{V}):=(r-1) c_{1}(\mathcal{V})^{2}-2 r c_{2}(\mathcal{V})
$$

where $c_{i}(\mathcal{V}), i=1,2$ are the Chern classes of $\mathcal{V}$. According to [5, Theorem p. 500], if $\mathcal{V}$ is $H$-stable for any ample line bundle $H$ on $S$, then $\delta(\mathcal{V})<0$ (Bogomolov inequality). Then $\mathcal{V}$ is said to be $B$-unstable if $\delta(\mathcal{V})>0$; consequently, in accordance with the usual terminology, we say that $\mathcal{V}$ is $B$-semistable if $\delta(\mathcal{V}) \leq 0, B$-stable if this is a strict inequality, and properly $B$-semistable if equality occurs.

\section{The canonical equation of $\Gamma$ for scrolls over surfaces}

Let $(X, L)$ be a polarized manifold of dimension $n$ which is a scroll over a smooth surface $S$, with projection $\pi: X \rightarrow S$. In particular, $X$ is a $\mathbb{P}^{n-2}$-bundle over $S$, hence $\chi\left(\mathcal{O}_{X}\right)=$ $\chi\left(\mathcal{O}_{S}\right)$. Set $\mathcal{E}=\pi_{*} L$; then $\mathcal{E}$ is an ample vector bundle of rank $n-1$ on $S$, and $X=\mathbb{P}(\mathcal{E})$, with tautological line bundle $L$. Since $\operatorname{rk}(\mathcal{E})=n-1$, and $\operatorname{dim} S=2$, the Chern-Wu relation says that

$$
L^{n-1}-\pi^{*} c_{1}(\mathcal{E}) \cdot L^{n-2}+\pi^{*} c_{2}(\mathcal{E}) \cdot L^{n-3}=0
$$

(see e.g., [6, p. 429]). This gives

$$
L^{n-1} \cdot \pi^{*} D=L^{n-2} \cdot \pi^{*}\left(D \cdot c_{1}(\mathcal{E})\right)=D \cdot c_{1}(\mathcal{E})
$$

for any line bundle $D$ on $S$. Recalling the canonical bundle formula

$$
K_{X}=-(n-1) L+\pi^{*}\left(K_{S}+c_{1}(\mathcal{E})\right)
$$

(3) allows us to compute all pluridegrees $d_{i}=K_{X}^{i} \cdot L^{n-i}(i=0, \ldots, n)$ of $(X, L)$. Clearly, $d=d_{0}=L^{n}$ is the degree of $(X, L)$. In particular, we get

$$
\begin{gathered}
d_{n}=K_{X}^{n}=(-1)^{n}(n-1)^{n-1}\left(\frac{n}{2} K_{S}^{2}+\left(\frac{n}{2}-1\right) c_{1}(\mathcal{E})^{2}-(n-1) c_{2}(\mathcal{E})\right) \\
d_{1}=K_{X} \cdot L^{n-1}=K_{S} \cdot c_{1}(\mathcal{E})-(n-2) c_{1}(\mathcal{E})^{2}+(n-1) c_{2}(\mathcal{E})
\end{gathered}
$$

and

$$
d=L^{n}=c_{1}(\mathcal{E})^{2}-c_{2}(\mathcal{E})
$$

Now let $\Gamma=\Gamma_{(X, L)}$ be the Hilbert curve of our scroll $(X, L)$. According to $(2)$ we set

$$
\delta:=\delta(\mathcal{E})=(n-2) c_{1}(\mathcal{E})^{2}-2(n-1) c_{2}(\mathcal{E}) .
$$

The following result extends [9, Proposition 2.1] to any dimension, providing the explicit canonical equation of $\Gamma$. 
Theorem 3.1 Let $(X, L)$ be an $n$-dimensional scroll over a smooth surface $S$, let $\mathcal{E}:=$ $\pi_{*} L$, where $\pi: X \rightarrow S$ is the scroll projection, and let $\delta$ be as in (7). Then the Hilbert curve $\Gamma$ of $(X, L)$ has the following canonical equation in terms of coordinates $(u, v)$

$$
p\left(\frac{1}{2}+u, v\right)=\left(\alpha u^{2}+\beta u v+\gamma v^{2}+\varepsilon\right) \prod_{i=1}^{n-2}\left((n-1) u-v+\frac{1}{2}(n-1-2 i)\right),
$$

where $\alpha, \beta, \gamma$, and $\varepsilon$ are given by the following expressions:

$$
\begin{gathered}
\alpha=\frac{(-1)^{n}}{(n-2) !} \frac{1}{2}\left(K_{S}^{2}+\frac{\delta}{n}\right), \\
\beta=\frac{2(-1)^{n}}{(n-2) !} \frac{1}{2}\left(K_{S} \cdot \frac{c_{1}(\mathcal{E})}{n-1}-\frac{\delta}{n(n-1}\right), \\
\gamma=\frac{(-1)^{n}}{(n-2) !} \frac{1}{2}\left(\frac{c_{1}(\mathcal{E})^{2}}{(n-1)^{2}}+\frac{\delta}{n(n-1)^{2}}\right), \\
\varepsilon=\frac{(-1)^{n}}{(n-2) !} \frac{1}{2}\left(2 \chi\left(\mathcal{O}_{S}\right)-\frac{K_{S}^{2}}{4}-\frac{\delta}{4 n}\right) .
\end{gathered}
$$

Proof. Recalling [4, Theorem 6.5] we know that the canonical equation of $\Gamma$ has an expression of the following type

$$
p(x, y)=R(x, y) \prod_{i=1}^{n-2}((n-1) x-y-i)=0
$$

where $R$ is a polynomial of degree 2 . Moreover, due to the symmetry properties of $\Gamma$, by using coordinates $(u, v)=\left(x-\frac{1}{2}, y\right)$ with the origin at the center of the involution induced by Serre duality, we can write

$$
R\left(\frac{1}{2}+u, v\right)=\alpha u^{2}+\beta u v+\gamma v^{2}+\varepsilon .
$$

To determine the coefficients $\alpha, \beta$, and $\gamma$ we proceed as in [7, Proposition 2.1]. Let $p_{0}(x, y, z)$ be the homogeneous polynomial associated with $p$. Since

$$
p(x, y)=\left(\alpha\left(x-\frac{1}{2}\right)^{2}+\beta\left(x-\frac{1}{2}\right) y+\gamma y^{2}+\varepsilon\right) \prod_{i=1}^{n-2}((n-1) x-y-i),
$$

evaluating $p_{0}$ on the line at infinity we get

$$
\begin{aligned}
p_{0}(x, 1,0)= & \left(\alpha x^{2}+\beta x+\gamma\right)((n-1) x-1)^{n-2} \\
= & \left(\alpha x^{2}+\beta x+\gamma\right)\left[(n-1)^{n-2} x^{n-2}-\ldots\right. \\
& \left.\cdots+(-1)^{n-3}\left(\begin{array}{l}
n-2 \\
n-3
\end{array}\right)(n-1) x+(-1)^{n-2}\right] \\
= & \alpha(n-1)^{n-2} x^{n}+\cdots+(-1)^{n}(\beta-(n-1)(n-2) \gamma) x+(-1)^{n} \gamma
\end{aligned}
$$


On the other hand, $\chi(M)=\frac{1}{n !} M^{n}+\ldots$ for any line bundle $M$ on $X$, where dots stand for lower degree terms, hence

$$
p_{0}(x, 1,0)=\frac{1}{n !}\left(x K_{X}+L\right)^{n}=\frac{1}{n !}\left[d_{n} x^{n}+\cdots+\left(\begin{array}{c}
n \\
n-1
\end{array}\right) d_{1} x+d\right] .
$$

For every power of $x$ we can thus equate the coefficients in the two expressions above. In particular, looking at the terms of degrees $n, 1$ and 0 , we get the following equalities

$$
\begin{gathered}
\alpha=\frac{1}{n !} \frac{1}{(n-1)^{n-2}} d_{n}, \\
\beta=\frac{(-1)^{n}}{n !}\left((n-1)(n-2) d+n d_{1}\right), \\
\gamma=\frac{(-1)^{n}}{n !} d .
\end{gathered}
$$

It remains to determine $\varepsilon$. Recalling that $\chi\left(\mathcal{O}_{X}\right)=\chi\left(\mathcal{O}_{S}\right)$, we get

$$
\chi\left(\mathcal{O}_{S}\right)=p(0,0)=\left(\frac{\alpha}{4}+\varepsilon\right) \prod_{i=1}^{n-2}(-i)=(-1)^{n-2}(n-2) !\left(\frac{\alpha}{4}+\varepsilon\right),
$$

and by using 13 this gives

$$
\varepsilon=\frac{(-1)^{n}}{n !}\left(n(n-1) \chi\left(\mathcal{O}_{S}\right)+\frac{(-1)^{n-1}}{4(n-1)^{n-2}} d_{n}\right) .
$$

Finally, taking into account (4), (5), (6), and (7), the above expressions can be rewritten as in (9), 110), (11), and (12), respectively.

Q.E.D.

In particular, we see that $\Gamma$ consists of

a) $n-2$ parallel lines of slope $n-1$, evenly spaced with step 1 on the $v$-axis, arranged symmetrically with respect to the origin, and

b) a conic $G$, also symmetric with respect to the origin.

This fact was already known from [4, Theorem 6.5]. The crucial point is that Theorem 3.1 provides an explicit equation for $G$. Actually, up to the multiplicative constant $\frac{(-1)^{n}}{(n-2) !}$, the conic $G$ is represented by the equation

$$
\frac{1}{2}\left[\begin{array}{lll}
u & v & 1
\end{array}\right] A_{\delta}\left[\begin{array}{l}
u \\
v \\
1
\end{array}\right]=0
$$

where

$$
A_{\delta}=\left[\begin{array}{ccc}
K_{S}^{2}+\frac{\delta}{n} & K_{S} \cdot \frac{c_{1}(\mathcal{E})}{(n-1)}-\frac{\delta}{n(n-1)} & 0 \\
K_{S} \cdot \frac{c_{1}(\mathcal{E})}{n-1}-\frac{\delta}{n(n-1)} & \frac{c_{1}(\mathcal{E})^{2}}{(n-1)^{2}}+\frac{\delta}{n(n-1)^{2}} & 0 \\
0 & 0 & 2 \chi\left(\mathcal{O}_{S}\right)-\frac{K_{S}^{2}}{4}-\frac{\delta}{4 n}
\end{array}\right]
$$




\section{$4 G$ itself as a Hilbert curve}

Referring to [4, Problem 6.6], and taking into account [7, Remark 4.1] and [9, Section 3], it is natural to ask the following question.

Is $G$ the Hilbert curve of $S$ for some $\mathbb{Q}$-polarization related to $(X, L)$ ?

The answer is negative in general. In fact it may even happen that there exists no $\mathbb{Q}$ polarized surface having $G$ as Hilbert curve. This is the case, for instance, for the scroll over $\mathbb{P}^{2}$ defined by $\mathcal{E}=\mathcal{O}_{\mathbb{P}^{2}}(1)^{\oplus(n-2)} \oplus \mathcal{O}_{\mathbb{P}^{2}}(2)$; a direct check mimicking [9, Proof of Proposition 3.1] shows that for no $n \geq 3$ there can exist a $\mathbb{Q}$-polarized surface $(\Sigma, \mathcal{M})$ such that $G=\Gamma_{(\Sigma, \mathcal{M})}$.

Coming back to $S$, let $\mathcal{L}$ be any ample $\mathbb{Q}$-line bundle. According to what we said in Section 2, the canonical equation of the Hilbert curve $\Gamma_{(S, \mathcal{L})}$ is

$$
p_{(S, \mathcal{L})}\left(\frac{1}{2}+u, v\right)=\frac{1}{2}\left[\begin{array}{lll}
u & v & 1
\end{array}\right] A^{\prime}\left[\begin{array}{l}
u \\
v \\
1
\end{array}\right]=0
$$

where

$$
A^{\prime}=\left[\begin{array}{ccc}
K_{S}^{2} & K_{S} \cdot \mathcal{L} & 0 \\
K_{S} \cdot \mathcal{L} & \mathcal{L}^{2} & 0 \\
0 & 0 & 2 \chi\left(\mathcal{O}_{S}\right)-\frac{K_{S}^{2}}{4}
\end{array}\right]
$$

Thus (15) has a positive answer if and only if there exists a nonzero constant factor $\rho \in \mathbb{Q}$ such that $A_{\delta}=\rho A^{\prime}$ for some $\mathbb{Q}$-ample line bundle $\mathcal{L}$ on $S$. In view of (14) this translates into the following conditions:

$$
\begin{gathered}
K_{S}^{2}+\frac{\delta}{n}=\rho K_{S}^{2}, \\
K_{S} \cdot \frac{c_{1}(\mathcal{E})}{n-1}-\frac{\delta}{n(n-1)}=\rho K_{S} \cdot \mathcal{L}, \\
\frac{c_{1}(\mathcal{E})^{2}}{(n-1)^{2}}+\frac{\delta}{n(n-1)^{2}}=\rho \mathcal{L}^{2}, \\
2 \chi\left(\mathcal{O}_{S}\right)-\frac{K_{S}^{2}}{4}-\frac{\delta}{4 n}=\rho\left(2 \chi\left(\mathcal{O}_{S}\right)-\frac{K_{S}^{2}}{4}\right) .
\end{gathered}
$$

Let's point out that $\rho$ must be positive. This follows from (19) because, recalling (6) and (7), we get

$$
\rho=\frac{2 d}{n(n-1) \mathcal{L}^{2}}>0 .
$$

Note also that (17) and (20) depend only on $S$, not involving $\mathcal{L}$; moreover, 17 can be rewritten as

$$
n(\rho-1) K_{S}^{2}=\delta
$$

and this shows that

$$
\delta=0 \text { if and only if either } \rho=1 \text { or } K_{S}^{2}=0 .
$$

Furthermore, in view of (17), condition (20) turns out to be equivalent to

$$
(\rho-1) \chi\left(\mathcal{O}_{S}\right)=0 .
$$

In case $\delta=0$, we can answer 15 in a precise way. 
Theorem 4.1 Let $(X, L)$ be a scroll over a smooth surface $S$ and let $\mathcal{E}=\pi_{*} L$, where $\pi: X \rightarrow S$ is the scroll projection. The conic $G$ is the Hilbert curve $\Gamma_{(S, \mathcal{L})}$ of $S$ endowed with an ample $\mathbb{Q}$-line bundle $\mathcal{L} \in \operatorname{Pic}(S) \otimes \mathbb{Q}$, HC-equivalent to the average polarization induced by $\mathcal{E}$, if and only if the vector bundle $\mathcal{E}$ is properly B-semistable, i. e., $\delta=0$.

Proof. Clearly, if $\delta=0$ then (14) shows that $A_{0}=A^{\prime}$ for $\mathcal{L}=\frac{c_{1}(\mathcal{E})}{n-1}$, the average polarization of $S$ induced by $\mathcal{E}$. More generally, the same is true for any $\mathbb{Q}$-polarization $\mathcal{L}$, HC equivalent to it. Thus $G=\Gamma_{(S, \mathcal{L})}$ for any such ample $\mathbb{Q}$-line bundle $\mathcal{L}$. To prove the converse, let $G=\Gamma_{(S, \mathcal{L})}$ for an ample $\mathbb{Q}$-line bundle $\mathcal{L}$, HC-equivalent to $\frac{1}{n-1} c_{1}(\mathcal{E})$. Then $\mathcal{L}^{2}=\frac{1}{(n-1)^{2}} c_{1}(\mathcal{E})^{2}$ and $K_{S} \cdot \mathcal{L}=K_{S} \cdot \frac{1}{n-1} c_{1}(\mathcal{E})$. Hence equations (19) and 18 become

$$
(\rho-1) c_{1}(\mathcal{E})^{2}=\frac{\delta}{n} \quad \text { and } \quad(\rho-1) K_{S} \cdot c_{1}(\mathcal{E})=-\frac{\delta}{n},
$$

respectively. Summing them up we get

$$
(\rho-1) c_{1}(\mathcal{E}) \cdot\left(K_{S}+c_{1}(\mathcal{E})\right)=0 .
$$

Now assume, by contradiction, that $\delta \neq 0$. Since $(17)-20)$ are satisfied, we see that $\rho \neq 1$. So 23) and (24) imply

$$
\chi\left(\mathcal{O}_{S}\right)=0 \quad \text { and } \quad c_{1}(\mathcal{E}) \cdot\left(K_{S}+c_{1}(\mathcal{E})\right)=0 .
$$

In particular, the former condition in (25) says that $S$ is not a rational surface. But this is not compatible with the latter condition, due to the following fact.

Lemma 4.2 Let $(X, L)$ be as in Theorem 4.1 and suppose that $(S, \mathcal{E}) \neq\left(\mathbb{P}^{2}, \mathcal{O}_{\mathbb{P}^{2}}(1)^{\oplus 2}\right)$. Then

$$
c_{1}(\mathcal{E}) \cdot\left(K_{S}+c_{1}(\mathcal{E})\right) \geq 0,
$$

with equality if and only if either $S=\mathbb{P}^{2}$ with $\mathcal{E}=\mathcal{O}_{\mathbb{P}^{2}}(1)^{\oplus 3}, \mathcal{O}_{\mathbb{P}^{2}}(1) \oplus \mathcal{O}_{\mathbb{P}^{2}}(2)$ or $T_{\mathbb{P}^{2}}$ (the tangent bundle), or $S=\mathbb{P}^{1} \times \mathbb{P}^{1}$ with $\mathcal{E}=\mathcal{O}_{\mathbb{P}^{1} \times \mathbb{P}^{1}}(1,1)^{\oplus 2}$. In particular, if $\chi\left(\mathcal{O}_{S}\right)=0$ then the above inequality is always strict.

Proof. Actually $K_{S}+c_{1}(\mathcal{E})$ is nef by [13, Theorem 2], due to the assumption. Hence the inequality follows from the ampleness of $c_{1}(\mathcal{E})$. Suppose it is an equality. Then the Hodge index theorem implies that $K_{S}+c_{1}(\mathcal{E}) \equiv 0$, because $\left(K_{S}+c_{1}(\mathcal{E})\right)^{2} \geq 0$, due to the nefness. Therefore $-K_{S} \equiv c_{1}(\mathcal{E})$ is ample, hence $S$ is a del Pezzo surface. This in turn implies that $-K_{S}=c_{1}(\mathcal{E})$, since $\operatorname{Pic}(S)$ has no torsion. Moreover $\left(S, c_{1}(\mathcal{E})\right)$ cannot contain lines since $\mathcal{E}$ is an ample vector bundle of rank $\geq 2$. Therefore $\left(S, c_{1}(\mathcal{E})\right)$ is either $\left(\mathbb{P}^{2}, \mathcal{O}_{\mathbb{P}^{2}}(3)\right)$ or $\left(\mathbb{P}^{1} \times \mathbb{P}^{1}, \mathcal{O}_{\mathbb{P}^{1} \times \mathbb{P}^{1}}(2,2)\right)$, by the classification of del Pezzo surfaces. Thus the assertion about $\mathcal{E}$ follows from the uniformity of $\mathcal{E}$ in view of a classical result of Van de Ven [10, p. 211] and its analogue for the quadric surface [12, Lemma 3.6.1]. Q.E.D.

This completes the proof of Theorem 4.1 .

Q.E.D.

(4.3.0) Case $\delta=0$ being settled, let's continue to explore what happens if $\delta \neq 0$. According to the above discussion, we know from $(22)$ and $(23)$ that

$$
\rho \neq 1, \quad K_{S}^{2} \neq 0 \quad \text { and } \quad \chi\left(\mathcal{O}_{S}\right)=0 .
$$

By the Enriques-Kodaira classification [1], the last condition in (26) implies that $S$ is birational to one of the following minimal surfaces: 
a) a $\mathbb{P}^{1}$-bundle over a smooth curve of genus one;

b) an abelian or a bielliptic surface;

c) an elliptic quasi-bundle in the sense of Serrano [11, Definition 1.2].

Note that $K_{S}^{2} \leq 0$ in all these cases, equality occurring if and only if $S$ is a minimal surface. Hence the second condition in $(26)$ becomes

$$
K_{S}^{2}<0 .
$$

Combining this with 22 , we get

Remark 1. Let $\delta \neq 0$; then $\delta$ and $1-\rho$ have the same sign.

Theorem 4.1 suggests that $\frac{1}{\mathrm{rk \mathcal {E }}}$ separates the $\mathbb{Q}$-line bundles $\mathcal{L}$ such that $G=\Gamma_{(S, \mathcal{L})}$ lying on the ray generated by $\operatorname{det} \mathcal{E}$, in terms of the B-stability properties of $\mathcal{E}$. Actually, arguing as in the proof of Theorem 4.1 and taking into account Remark 1, we can prove the following fact.

Proposition 4.3 Let $(X, L)$ be a scroll over a smooth surface $S$ and let $\mathcal{E}=\pi_{*} L$, where $\pi: X \rightarrow S$ is the scroll projection. Suppose that $G=\Gamma_{(S, \mathcal{L})}$ for an ample $\mathbb{Q}$-line bundle $\mathcal{L} \in \operatorname{Pic}(S) \otimes \mathbb{Q}, \mathrm{HC}$-equivalent to $\lambda c_{1}(\mathcal{E})$ for some positive $\lambda \in \mathbb{Q}$. Then $\mathcal{E}$ is B-semistable (B-unstable) if and only if $\lambda \leq \frac{1}{n-1}\left(\lambda>\frac{1}{n-1}\right)$.

Proof. Of course we can assume that $\delta \neq 0$ by Theorem 4.1. hence $\chi\left(\mathcal{O}_{S}\right)=0$ by 26 . Since $\mathcal{L}^{2}=\lambda^{2} c_{1}(\mathcal{E})^{2}$ and $K_{S} \cdot \mathcal{L}=\lambda K_{S} \cdot c_{1}(\mathcal{E})$ (19) and (18) give

$$
\left((n-1)^{2} \lambda^{2} \rho-1\right) c_{1}(\mathcal{E})^{2}=\frac{\delta}{n} \quad \text { and } \quad((n-1) \lambda \rho-1) K_{S} \cdot c_{1}(\mathcal{E})=-\frac{\delta}{n}
$$

respectively, and summing them up we get

$$
((n-1) \lambda \rho-1) c_{1}(\mathcal{E}) \cdot\left(K_{S}+c_{1}(\mathcal{E})\right)+(n-1) \lambda \rho((n-1) \lambda-1) c_{1}(\mathcal{E})^{2}=0 .
$$

Since $\lambda>0$, recalling (21) and Lemma 4.2 we thus see that $\rho-1<0$ if $\lambda>\frac{1}{n-1}$, while $\rho-1>0$ if $\lambda<\frac{1}{n-1}$. Then Remark 1 is enough to conclude.

Q.E.D.

Continuing the study of case $\delta \neq 0$, here we determine further explicit conditions on $(S, \mathcal{E})$ for being $G=\Gamma_{(S, \mathcal{L})}$. As already noted, the system of (17)-20) is equivalent to that of the first three equations only. Look at it as a system in the two unknowns $\rho$ and $\frac{\delta}{n}$. Clearing denominators we can rewrite it as

$$
\left\{\begin{array}{ccc}
K_{S}^{2} \rho-\frac{\delta}{n} & = & K_{S}^{2} \\
(n-1) K_{S} \cdot \mathcal{L} \rho+\frac{\delta}{n} & = & K_{S} \cdot c_{1}(\mathcal{E}) \\
(n-1)^{2} \mathcal{L}^{2} \rho-\frac{\delta}{n} & = & c_{1}(\mathcal{E})^{2} .
\end{array}\right.
$$

The augmented matrix of $[28)$, say $[\mathcal{A} \mid B], \mathcal{A}$ standing for the coefficient matrix, is:

$$
[\mathcal{A} \mid B]=\left[\begin{array}{ccc}
K_{S}^{2} & -1 & K_{S}^{2} \\
(n-1) K_{S} \cdot \mathcal{L} & 1 & K_{S} \cdot c_{1}(\mathcal{E}) \\
(n-1)^{2} \mathcal{L}^{2} & -1 & c_{1}(\mathcal{E})^{2}
\end{array}\right]
$$


Note that $\operatorname{rk}(\mathcal{A})=2$; actually the determinant of the submatrix consisting of the first and the third rows of $\mathcal{A}$ is

$$
\Delta=-K_{S}^{2}+(n-1)^{2} \mathcal{L}^{2}>0,
$$

by (27). Thus our system 28$]$ has a solution in $\mathbb{Q}^{2}$ if and only if

$$
\operatorname{det}[\mathcal{A} \mid B]=0 .
$$

This condition, however, does not take into account that $\delta$ must be an integer. In fact we will use it only as a necessary condition. By adding the second row to both the first and the third one of $[\mathcal{A} \mid B]$, we see that

$$
\begin{aligned}
\operatorname{det}[\mathcal{A} \mid B]= & \left|\begin{array}{ccc}
K_{S} \cdot\left(K_{S}+(n-1) \mathcal{L}\right) & 0 & K_{S} \cdot\left(K_{S}+c_{1}(\mathcal{E})\right) \\
(n-1) K_{S} \cdot \mathcal{L} & 1 & K_{S} \cdot c_{1}(\mathcal{E}) \\
(n-1) \mathcal{L} \cdot\left(K_{S}+(n-1) \mathcal{L}\right) & 0 & c_{1}(\mathcal{E}) \cdot\left(K_{S}+c_{1}(\mathcal{E})\right)
\end{array}\right| \\
= & \left(K_{S} \cdot\left(K_{S}+(n-1) \mathcal{L}\right)\right)\left(c_{1}(\mathcal{E}) \cdot\left(K_{S}+c_{1}(\mathcal{E})\right)\right) \\
& \quad-(n-1)\left(\mathcal{L} \cdot\left(K_{S}+(n-1) \mathcal{L}\right)\right)\left(K_{S} \cdot\left(K_{S}+c_{1}(\mathcal{E})\right)\right) \\
= & \left(K_{S}+(n-1) \mathcal{L}\right) \cdot\left(k K_{S}-(n-1) h \mathcal{L}\right),
\end{aligned}
$$

where $h:=K_{S} \cdot\left(K_{S}+c_{1}(\mathcal{E})\right)$ and $k:=c_{1}(\mathcal{E}) \cdot\left(K_{S}+c_{1}(\mathcal{E})\right)$. Therefore 30) is equivalent to

$$
k K_{S}^{2}+(k-h)(n-1) K_{S} \cdot \mathcal{L}-h(n-1)^{2} \mathcal{L}^{2}=0 .
$$

Remark 2. i) Note that $k>0$ in view of Lemma 4.2 , since $S$ is not rational, as $\chi\left(\mathcal{O}_{S}\right)=0$. ii) Moreover, $h<k$, since $h-k=\left(K_{S}-c_{1}(\mathcal{E})\right)\left(K_{S}+c_{1}(\mathcal{E})\right)=K_{S}^{2}-c_{1}(\mathcal{E})^{2}<0$ by (27). iii) We can assume that $K_{S}+c_{1}(\mathcal{E})$ is ample; otherwise $\left(S, c_{1}(\mathcal{E})\right)$ would be in a restricted list of cases that are not compatible with what we know about $S$ (e.g., see [2, Proposition 7.2.2 and Theorem 7.2.3]). So, if $h<0$ then no positive multiple of $K_{S}$ can be effective, and therefore $S$ is ruled in view of the Enriques theorem [1, Corollary VI.18]. Then, according to the possibilities listed in (4.3.0), $S$ is necessarily a non-minimal elliptic ruled surface. On the contrary, if $S$ is birational to either an abelian or a biellipic surface or to an elliptic quasi-bundle, then a positive multiple of $K_{S}$ is effective and nontrivial, since $S$ is non-minimal. Hence $h>0$.

iv) Consider the $\mathbb{Q}$-line bundle $T:=K_{S}-(n-1) \frac{h}{k} \mathcal{L}$. Condition 30 combined with the Hodge index theorem implies either $T \equiv 0$, or $T^{2}<0$. The former case cannot occur: otherwise it would be $K_{S} \equiv(n-1) \frac{h}{k} \mathcal{L}$, hence $K_{S}^{2}=(n-1)^{2}\left(\frac{h}{k}\right)^{2} \mathcal{L}^{2} \geq 0$, which contradicts (27). Therefore $T^{2}<0$. This, however, does not seem to have any further significant implication.

Provided that condition (31) is satisfied, the solution $\left(\rho, \frac{\delta}{n}\right)$ of $(28)$ is the same as that of the linear system consisting of the first and the third equations only. In particular, this gives

$$
\rho=\frac{1}{\Delta}\left|\begin{array}{cc}
K_{S}^{2} & -1 \\
c_{1}(\mathcal{E})^{2} & -1
\end{array}\right|=\frac{c_{1}(\mathcal{E})^{2}-K_{S}^{2}}{(n-1)^{2} \mathcal{L}^{2}-K_{S}^{2}}
$$


and

$$
\frac{\delta}{n}=\frac{1}{\Delta}\left|\begin{array}{cc}
K_{S}^{2} & K_{S}^{2} \\
(n-1)^{2} \mathcal{L}^{2} & c_{1}(\mathcal{E})^{2}
\end{array}\right|=K_{S}^{2} \frac{c_{1}(\mathcal{E})^{2}-(n-1)^{2} \mathcal{L}^{2}}{(n-1)^{2} \mathcal{L}^{2}-K_{S}^{2}}
$$

In particular, since $\delta$ and $1-\rho$ have the same sign (Remark 1 ), this says that

$$
\mathcal{E} \text { is B-stable if and only if } \rho>1 \text { if and only if } \mathcal{L}^{2}<\frac{1}{(n-1)^{2}} c_{1}(\mathcal{E})^{2}
$$

(B-instability is characterized by opposite inequalities).

Going back to (17), we know that $\rho=\frac{n K_{S}^{2}+\delta}{n K_{S}^{2}}$. According to 27) we can write $K_{S}^{2}=-t$, where $t$ is a positive integer representing the minimal number of blowing-ups a birational morphism from $S$ to its minimal model factors through. Hence

$$
\rho=\frac{n t-\delta}{n t} .
$$

By combining (34) with (32), we get

$$
\frac{n t-\delta}{n t}=\frac{t+c_{1}(\mathcal{E})^{2}}{t+(n-1)^{2} \mathcal{L}^{2}}
$$

Clearing denominators and recalling (7) and (6), this gives

$$
(n-1)^{2}(n t-\delta) \mathcal{L}^{2}=t\left(n c_{1}(\mathcal{E})^{2}+\delta\right)=2(n-1) d t .
$$

Similarly, combining (34) with (18) we get

$$
(n-1)(n t-\delta) K_{S} \cdot \mathcal{L}=t\left(n K_{S} \cdot c_{1}(\mathcal{E})-\delta\right) .
$$

Therefore,

$$
\mathcal{L}^{2}=\frac{2 d t}{(n-1)(n t-\delta)} \quad \text { and } \quad K_{S} \cdot \mathcal{L}=\frac{\left(n K_{S} \cdot c_{1}(\mathcal{E})-\delta\right) t}{(n-1)(n t-\delta)}
$$

where $d$ is the degree of $(X, L), \delta$ is given by (7), and $t=-K_{S}^{2}>0$. In particular, $\delta<n t$. We stress that the right hands in (37) are expressed only in terms of $(S, \mathcal{E})$. Finally, these values allow us to reformulate (31) in the following form

$$
n\left((k-h) K_{S} \cdot c_{1}(\mathcal{E})-k t\right)=h(2(n-1) d-\delta) .
$$

In conclusion, all conditions we obtained can be summarized as follows.

Proposition 4.4 Let $(X, L)$ be a scroll of degree $d$ over a smooth surface $S$, let $\mathcal{E}=\pi_{*} L$, where $\pi: X \rightarrow S$ is the scroll projection, suppose that $\mathcal{E}$ is not properly B-semistable and let $\delta$ be its Bogomolov number. Assume that the conic $G$ is the Hilbert curve $\Gamma_{(S, \mathcal{L})}$ of $S$ for some ample $\mathbb{Q}$-line bundle $\mathcal{L} \in \operatorname{Pic}(S) \otimes \mathbb{Q}$. Then $S$ is birational to a surface as in a), b) or c) in (4.3.0) and the number of blowing-ups necessary to obtain $S$ from its minimal model is $t>\min \left\{0, \frac{\delta}{n}\right\} ;$ moreover, $\mathcal{L}^{2}<\frac{1}{(n-1)^{2}} c_{1}(\mathcal{E})^{2}$ if and only if $\mathcal{E}$ is B-stable; furthermore, $\mathcal{L}^{2}$ and $K_{S} \cdot \mathcal{L}$ are expressed by (37), and condition (38) is satisfied. 


\section{References}

[1] A. Beauville, Complex Algebraic Surfaces, Cambridge Univ. Press, 1983 (translation of Surfaces algébriques complexes, Astérisque, 54 Soc. Math. France, Paris (1978)).

[2] M.C. Beltrametti and A.J. Sommese, The Adjunction Theory of Complex Projective Varieties, Expositions in Mathematics, vol. 16, de Gruyter, 1995.

[3] M.C. Beltrametti and A.J. Sommese, Comparing the classical and the adjunction theoretic definition of scrolls, Geometry of Complex Projective Varieties, Proceedings Cetraro, 1990, Seminars and Conferences, n. 9, Mediterranean Press, 1993, 55-74.

[4] M.C. Beltrametti, A. Lanteri, and A.J. Sommese, Hilbert curves of polarized varieties, J. Pure Appl. Algebra 214 (2010), 461-479.

[5] F.A. Bogomolov, Holomorphic tensors and vector bundles on projective manifolds, Izv. Akad. Nauk SSSR Ser. Mat. 42 (1978), 1227-1287 (Engl. Transl. Math. USSR-Izv. 13 (1979), 499555 .

[6] R. Hartshorne, Algebraic Geometry, Graduate Texts in Math., vol. 52, Springer-Verlag, New York, 1978.

[7] A. Lanteri, Characterizing scrolls via the Hilbert curves, Internat. J. Math. 25(11) (2014), [17 pages].

[8] A. Lanteri, HC-equivalence vs numerical equivalence for ample line bundles on surfaces, Rend. Circ. Mat. Palermo, II Ser, 66 (2017), 113-123.

[9] A. Lanteri, Hilbert curves of 3-dimensional scrolls over surfaces, J. Pure Appl. Algebra 222 (2018), 139-154.

[10] C. Okonek, M. Schneider, and H. Spindler, Vector Bundles on Complex Projective Spaces, Progr. Math., vol. 3, 1980, Birkhäuser, Boston.

[11] F. Serrano, Fibrations on algebraic surfaces, Geometry of Complex Projective Varieties, Proceedings Cetraro, 1990, Seminars and Conferences, n. 9, Mediterranean Press, 1993, 289-301.

[12] J. Wiśniewski, Length of extremal rays and generalized adjunction. Math. Z. 200 (1989), 409-427.

[13] Y-G. Ye and Q. Zhang, On ample vector bundles whose adjunction bundles are not numerically effective. Duke Math. J. 60 (1990), 671-687.

A. Lanteri

Dipartimento di Matematica "F. Enriques"

Università degli Studi di Milano

Via C. Saldini, 50

I-20133 Milano, Italy.

e-mail antonio.lanteri@unimi.it 